\title{
Consultoria psiquiátrica em hospital geral: inviável ou promissora?
}

\section{Consultation-Liaision psychiatry: impracticable or promising?}

Neury José Botega ${ }^{\mathrm{a}}$, Luiz Gustavo Guilhermano ${ }^{\mathrm{b}}$, Renato Michel ${ }^{\mathrm{c}}$, Celso Garcia Jr. ${ }^{\mathrm{d}}$, Fabiana G Machado ${ }^{\mathrm{b}}$, Fernanda Crestana $^{\mathrm{b}}$, Jacqueline Balestrin ${ }^{\mathrm{b}}$ e Luciana Frantz

aDepartamento de Psicologia Médica e Psiquiatria da FCM Unicamp, bepartamento de Psiquiatria da Faculdade de Medicina da PUC/RS (FMPUC/RS), 'Universidade de Santa Cruz do Sul (UNISC), 'Serviço de Transplante de Medula Óssea do HC/Unicamp

Resumo Objetivo: Avaliar alguns fatores que influenciam a prática de interconsulta (IC).

Métodos: Todos os 64 psiquiatras formados nos programas de residência médica da PUC/RS (1977-1995) e 52 formados na Unicamp (1986-1995) foram selecionados para participar de uma entrevista por telefone sobre os seguintes itens: dados sociodemográficos, principais atividades profissionais, características do trabalho em IC (tipo de serviço, carga horária, características do atendimento, qualificação profissional, motivações e dificuldades).

Resultados: Obtiveram-se 106 (91\%) entrevistas, sendo 51\% dos entrevistados de sexo masculino. Um terço teve experiência com IC apenas durante a residência, 37\% trabalham atualmente nessa área e 30\% já trabalharam em IC após a residência, mas haviam deixado de fazê-lo. Dentre os que trabalham atualmente em IC, há predomínio do sexo masculino (qui-quadrado=6,65; $\mathrm{p}=0,03)$, tanto na população formada na PUC/RS $(65 \%)$, quanto na Unicamp (63\%). Apenas $6 \%$ apontam falta de treinamento adequado entre as razões que pesaram na desistência de trabalhar em IC. As principais dificuldades referiram-se à escassez e à imprevisibilidade do tempo despendido, à baixa remuneração e à pouca valorização de parte dos colegas médicos.

Conclusões: Considerável proporção dos psiquiatras entrevistados continua trabalhando em IC (37\%). Apesar de considerarem adequado o treinamento recebido durante a residência, apontaram as principais dificuldades encontradas nessa tarefa: tempo, dinheiro e valorização do trabalho realizado.

Descritores Interconsulta. Consultoria psiquiátrica. Hospital Geral.

Abstract Objective: Factors influencing the practice of psychiatric consultation-liaison (CL) were analyzed.

Methods: All 116 psychiatrists trained in two Brazilian medical schools were contacted by telephone, invited to participate in the study and then interviewed.

Results: 106 (91\%) doctors were interviewed; 51\% were male. One third had carried out CL only during psychiatric training, 37\% were working with CL at the time of the interview, and $30 \%$ had had experience with $\mathrm{CL}$ after the psychiatric training, but stopped afterward. Among those working with CL, there was a prevalence of men $(64 \%$, chi-square $=6.65 ; \mathrm{p}=0.03)$. Only $6 \%$ mentioned inadequate training as one of the reasons for not pursuing working with CL. The most frequently reported difficulties were: the amount of time needed, the unpredictable nature of the psychiatric consultation requests, low pay for the work, and lack ofappraisal of CL practiceby other medical colleagues.

Conclusions: $37 \%$ of the interviewed doctors worked with CL. In spite of the adequate training received during medical residency, they had to cope with their lack of time, low pay, and their colleagues' low appraisal of CL.

Keywords Psychiatric consultation. General Hospital. Health personnel.

\section{Introdução}

Nas duas últimas décadas, serviços de consultoria e de ligação abriram caminho para a instalação da psiquiatria em vários hospitais gerais universitários. ${ }^{1}$ Os que trabalham na área, po- rém, têm-se questionado em que resultam os esforços para ensinar interconsulta (IC) quando o residente de psiquiatria deixa a instituição. Há mais de duas décadas, Lipowski observava, com preocupação, que poucos psiquiatras permaneciam no tra- 
balho de consultoria por mais do que alguns anos. ${ }^{2}$ Entre nós, Guilhermano et al referiram-se a uma baixa natalidade de novos psiquiatras interconsultores e a uma alta mortalidade entre seus praticantes veteranos. ${ }^{3}$

Duas instituições brasileiras que mantêm programas de residência médica em psiquiatria, com forte tradição no ensino de IC, 45 aproximaram-se para a realização dessa pesquisa: a Faculdade de Medicina da Pontifícia Universidade Católica do Rio Grande do Sul (PUC/RS), localizada em Porto Alegre, e a Faculdade de Ciências Médicas da Universidade Estadual de Campinas (Unicamp). Este artigo tem o objetivo de avaliar alguns fatores que influenciam a prática da IC, segundo opinião de psiquiatras formados nos programas de residência médica em psiquiatria dessas instituições.

\section{Métodos}

Delineou-se um inquérito populacional, baseado em entrevista realizada por telefone. Partiu-se de listagens de todos os psiquiatras formados na PUC/RS, de 1977 a 1995 (n=64) e na Unicamp, entre 1986 e $1995(\mathrm{n}=52)$.

Uma entrevista foi desenvolvida conjuntamente pelas equipes das duas instituições. Tomando por base os resultados de um estudo-piloto, realizado com dez psiquiatras formados nos últimos dois anos, definiram-se detalhes do instrumento e se estabeleceu a maneira padronizada de aplicá-lo, tarefa que foi facilitada pelo formato "fechado" de respostas. Em sua versão final, a entrevista levou 10-15 minutos para ser aplicada e continha as seguintes seções: (a) dados sociodemográficos; (b) principais atividades profissionais; e (c) características do trabalho em IC (tipo de serviço, carga horária, características do atendimento, qualificação profissional, motivações, dificuldades e, quando pertinente, razões para desistir de trabalhar em IC). Os dados obtidos foram analisados por estatística descritiva.

\section{Resultados}

Todos os psiquiatras contatados concordaram em participar. Da PUC/RS, obtiveram-se 55 (86\%) entrevistas, e da Unicamp, $51(98 \%)$, perfazendo um total de 106 participantes. Não foram realizados dez contatos: três por causa de falecimento e sete por falta de um endereço atualizado. Por razões didáticas, quando houver semelhança no que se obteve nas duas instituições, os dados serão apresentados conjuntamente.

Um número semelhante de homens e de mulheres formou-se em psiquiatria (PUC/RS: 27 e 28; Unicamp: 27 e 24, respectivamente). Dentre os 39 (37\%) psiquiatras que trabalham atualmente em IC, há predomínio do sexo masculino, tanto na população formada na PUC/RS (65\%), quanto na Unicamp (63\%) (Tabela). Desses profissionais, $43 \%$ encontram-se vinculados a uma equipe de IC, 79\% realizam tão somente consultoria, apenas $8 \%$ fazem ligação e $18 \%$ estão ou estiveram vinculados a programas de pós-graduação. Trabalha-se nessa atividade em média 7 horas por semana. No entanto, apenas $30 \%$ cumprem horário fixo.

Dentre as principais motivações para trabalhar em IC, encontram-se: (a) "vocacional" (61\%); (b) "renovação de conhe-
Tabela - Atividades na área de Interconsulta (IC) em relação ao sexo dos participantes $(n=106)$.

\begin{tabular}{lllr}
\hline & $\begin{array}{l}\text { Realiza } \\
\text { IC atualmente }\end{array}$ & $\begin{array}{l}\text { Realizou IC após } \\
\text { residência, mas } \\
\text { deixou de fazê-lo }\end{array}$ & $\begin{array}{r}\text { Realizou IC } \\
\text { somente durante } \\
\text { a residência }\end{array}$ \\
$\begin{array}{l}\text { Sexo } \\
\text { Masculino }\end{array}$ & $\begin{array}{l}\text { PUC/RS }=13 \\
\text { Unicamp }=12\end{array}$ & $\begin{array}{l}\mathrm{PUC} / \mathrm{RS}=11 \\
\text { Unicamp }=6\end{array}$ & $\begin{array}{r}\mathrm{PUC} / \mathrm{RS}=3 \\
\text { Unicamp }=9\end{array}$ \\
$\begin{array}{l}\text { Sexo } \\
\text { Feminino }\end{array}$ & 14 & 15 & 23 \\
& $\begin{array}{l}\text { PUC/RS }=7 \\
\text { Unicamp }=7\end{array}$ & $\begin{array}{l}\mathrm{PUC} / \mathrm{RS}=6 \\
\text { Unicamp }=9\end{array}$ & $\begin{array}{r}\mathrm{PUC} / \mathrm{RS}=15 \\
\text { Unicamp }=8\end{array}$ \\
\hline Teste qui-quadrado $=6,65 ; 2$ graus de liberdade; $\mathrm{p}=0,03$ &
\end{tabular}

cimentos" (51\%); (c) "expansão do relacionamento profissional" (43\%); (d) "acadêmica/científica" (36\%); (e) "forma de contrapor o isolamento profissional do consultório" (33\%); e (f) "financeira" (7\%).

As dificuldades mais citadas pela maioria dos entrevistados foram: (a) "irregularidade das chamadas" (33\%); (b) "desvalorização do psiquiatra pelos outros médicos" (33\%); (c) "remuneração insuficiente" (25\%); (d) "ambiente de trabalho adverso" (20\%); e (e) "desvalorização das atividades por outros psiquiatras" (20\%). A questão que investigava alguma forma de "desgaste físico ou emocional" decorrente da atividade em interconsulta provocou $50 \%$ de respostas afirmativas entre os entrevistados da PUC/RS e $37 \%$ entre os da Unicamp.

Há um grupo que já trabalhou em IC após a residência, mas não mais trabalhava à época deste estudo, composto por 32 (30\%) psiquiatras. Metade desses profissionais trabalhou pelo menos quatro anos em IC. Dentre os principais motivos da desistência de trabalhar na área estão (a) "falta de tempo" (47\%); (b) "honorários insuficientes" (28\%); (c) "falta de oportunidade de trabalho na área" (28\%); e (d) "preferência pelo trabalho individual com o paciente" (15\%). Dentre os que pararam de trabalhar em IC, apenas $6 \%$ apontam a falta de treinamento adequado entre as razões que pesaram na desistência. Em relação ao tempo de formado, os psiquiatras distribuíram-se eqüitativamente entre os grupos.

\section{Discussão}

Decorridos alguns anos do término da residência, 37\% dos psiquiatras formados na PUC/RS e na Unicamp continuam trabalhando em interconsulta. Isso não nos parece pouco. É preciso lembrar, todavia, que se trata dos dois Estados com os maiores números de unidades de psiquiatria em hospitais gerais $^{6}$ e, particularmente, de duas instituições com forte tradição no ensino de IC. Quando se pensa em Brasil, isso deve ser relativizado. Dados coletados há dez anos demonstraram que, apesar de o serviço de IC estar disponível em 90\% das residências médicas em psiquiatria, em apenas metade havia um estágio estruturado para se treinar o residente nessa atividade?

Nas duas instituições encontraram-se dados semelhantes: escassez de tempo, de dinheiro e de valorização do trabalho realizado são as principais dificuldades nesse campo de atividade. Diferenças nos perfis dos participantes das duas 
instituições não redundaram em variações apreciáveis nos dois conjuntos de respostas no tocante às opiniões, motivações e dificuldades em relação à interconsulta. Essa ausência de discrepância dá mais força aos achados do presente trabalho.

As informações obtidas do grupo que segue fazendo interconsulta contribuem para a montagem de um panorama dessa subespecialidade. Trata-se de um profissional que se sente motivado por sua vocação, bem como pela possibilidade de renovar conhecimentos e de expandir seu relacionamento profissional. Em pouco mais da metade das vezes, realiza-se um trabalho solitário, de 7 horas semanais em média, sem horário fixo, que responde a solicitações feitas por colegas. Não ter vinculação com uma equipe de IC e fazer consultoria esporadicamente, significa entrar em campo só para atender casos especiais, sem o compromisso com a manutenção do serviço. Isso torna crítica a qualidade de seu trabalho.

A questão do gênero relacionada às atividades de IC parece um campo aberto para investigações. Supõe-se que as mulheres psiquiatras entrevistadas, em grande proporção, necessitam de horários esquematizados, de modo a permitir o desempenho de tarefas ligadas ao papel de mãe. Provavelmente, isso faz com que prefiram desempenhar atividades onde haja relação direta entre horas trabalhadas e reembolso financeiro, o que não tem sido até a atualidade uma característica da IC. Devido ao caráter restrito do questionário, bem como à carência de dados a respeito da distribuição por sexo dos médicos que trabalham em hospitais gerais, torna-se difícil ir além nessa formulação.

Os conflitos entre identidade e papel profissionais podem crescer a tal ponto, que desencorajam a continuidade do trabalho em IC. Há no interconsultor a percepção de que seu trabalho não é adequadamente valorizado por outros especialistas (1/3 das respostas) e mesmo por colegas da especialidade (1/5 das respostas). Há, também o risco de esgotamento (burnout) observado entre interconsultores psiquiátricos ${ }^{8} \mathrm{e}$ relatado por $43 \%$ dos entrevistados. Não prevista pelo SUS, nem por vários convênios, a IC é desconsiderada, o que obriga oferecer "amostras grátis" deste trabalho, na aposta de que sejam percebidas as vantagens de sua utilização.

\title{
Referências
}

1. Nogueira-Martins LA, Botega NJ. Interconsulta psiquiátrica no Brasil: desenvolvimentos recentes. Rev ABP-APAL 1998;20:105-11.

2. Lipowski Z. Consultation-liaison psychiatry: na overview. Am J Psychiatry 1974; 131:623-30.

3. Guilhermano LG, Machado FG, Crestana F, Balestrin J, Frantz L. Consultoria-liaison psiquiátrica em hospital geral. Rev Psiquiatr Consiliar Ligação 1998;4:17-31.

4. Cataldo Neto A, Machado FG, Crestana F, Balestrin JÁ, Frantz L, Michel R. Duas décadas de formação psiquiátrica na PUC/RS: perfil dos psiquiatras formados entre 1976 e 1994. Rev Psiquiatr RS 1998;17:201-9.

5. Laurito Jr JB, Botega NJ. HC/Unicamp: programa de treinamento em interconsulta psiquiátrica. In: Miguel Filho EC, Ramadam ZBA, Marbergier A, Sousa DG, editores. Interconsulta psiquiátrica no Brasil. São Paulo: Astúrias; 1990. p. 115-21.

6. Botega NJ, Schechtman A. Censo nacional de unidades de psiquiatria em hospitais gerais: I. Situação atual e tendências. Rev ABP-APAL 1997;19:79-86.

7. Botega NJ. Consultation-liaison psychiatry in Brazil: psychiatric residency training. Gen Hosp Psychiat 1992;14:186-91.

8. Wise TN, Berlin RM. Burnout: stresses in consultation-liaison psychiatry. Psychosom 1980;21:205-12.

\author{
Correspondência \\ Neury José Botega \\ Depto. de Psiquiatria \\ FCM/ Unicamp \\ 13081-970 Caixa Postal 6111, Campinas, SP \\ Tel.: (0xx19) 289-4819 \\ E-mail: botega@fcm.unicamp.br
}

